\title{
ON THE DISTANCE TO CYGNUS X-1 (HDE 226868)*
}

\author{
JESSE BREGMAN, DENNIS BUTLER, EDWARD KEMPER, ALAN KOSKI \\ R. P. KRAFT, and R. P. S. STONE** \\ Lick Observatory, Board of Studies in Astronomy and Astrophysics, \\ University of California, Santa Cruz, Calif., U.S.A.
}

\begin{abstract}
From $U, B, V$ photometry of 104 stars in a field of radius $\sim 30^{\prime}$ centered on the X-ray binary star Cyg X-1 (HDE 226868), we have studied the color excess $E(B-V)$ as a function of distance. Spectral types were observed de novo for 42 of these stars. We conclude that HDE 226868 cannot be nearer than $1 \mathrm{kpc}$, and is probably at a distance of $2.5 \mathrm{kpc}$ or more. The primary component is therefore a luminous $\mathrm{OB}$ star of mass $\sim 30 \mathfrak{M}_{\odot}$, and the $\mathrm{X}$-ray component has a minimum mass $\sim 6 \mathfrak{M}_{\odot}$.
\end{abstract}

* Contributions from the Lick Observatory No. 385, Astrophys. J. Letters 185, L117, November 15, 1973.

** Supported in part by NSF Science Departmental Improvement Grant GU-3162. 